\title{
Correlation Between the FWHM of the Broad Emission Lines and the Soft X-Ray Photon Index in the PG Quasars
}

\author{
P. M. Gondhalekar, ${ }^{1}$ C. Rouillon-Foley, ${ }^{2}$ and B. J. Kellett ${ }^{1}$ \\ ${ }^{1}$ Rutherford Appleton Laboratory, Chilton, OXON, OX11 OQX, UK \\ ${ }^{2}$ Mullard Space Science Laboratory, University College London, \\ Holmbury St. Mary, Dorking, Surrey, RH5 6NT, UK
}

\begin{abstract}
The correlation between the FWHM of Ly $\alpha, \mathrm{CIV}$ and $\mathrm{H} \beta$ lines and the photon index of the soft X-ray continuum is investigated for a sample of PG quasars. Only the FWHM of $\mathrm{H} \beta$ line is correlated with the photon index and this correlation depends on the sample of the AGNs considered.
\end{abstract}

\section{Introduction}

The correlation between the emission-line parameters and the continuum parameters in Seyfert 1 galaxies and quasars can provide fundamental insight into the geometry and the kinematics of the line-forming region (the BLR) and the continuum-emitting region. In this paper, the correlations between the FWHM of Ly $\alpha, \mathrm{C} I V$, and $\mathrm{H} \beta$ lines and the photon index $\Gamma$ of the soft $\mathrm{X}$-ray continuum $(0.2-2.0 \mathrm{keV})$ are investigated. This analysis is restricted to a sample of quasars drawn from the PG survey of quasars in order to minimize any bias which may be introduced by selection.

The FWHM of Ly $\alpha$ and $\mathrm{C}$ IV lines were obtained from IUE observations and the accuracy of these widths is about $10 \%$. The FWHM of $\mathrm{H} \beta$ line was obtained from Boroson \& Green (1992). The ROSAT PSPC data on this sample of quasars were obtained from the ROSAT Archive at the University of Leicester and analyzed with the ASTERIX X-ray data processing package (Version 17a) provided by the STARLINK Project. The data were deconvolved with a single absorbed power-law and the Galactic column density was left a free parameter (Gondhalekar, Rouillon-Foley, \& Kellett 1996a,b).

\section{Correlation Between the FWHM and the Photon Index}

To investigate the correlation between FWHM and $\Gamma$ a straight line of the form FWHM $=\left(a \pm \sigma_{a}\right)+\left(b \pm \sigma_{b}\right) \Gamma$ was fit to the data for the three emission lines. The slope of the straight line fit, the Spearman rank correlation coefficient (SR) and the two-tailed probability $(P)$ that the correlation could not arise by chance are given in Table 1. There is no correlation between FWHM and $\Gamma$ for Ly $\alpha$ and $\mathrm{C}$ IV lines. However, there is a strong correlation between FWHM and $\Gamma$ for the $\mathrm{H} \beta$ line. This correlation was first pointed out by Laor et al. (1994) and has 
since been investigated by Boller et al. (1996) for narrow-line Seyfert galaxies and by Wang et al. (1996) for a heterogeneous sample of Seyfert 1 galaxies and quasars. We would like to point out that there is no correlation between FWHM and $\Gamma$ for narrow-line Seyfert galaxies, the parameters of a straight line fit to the data of Boller et al. are also given in Table 1. Similarly, the slope of the correlation for the heterogeneous sample of Wang et al. is significantly higher than that obtained for the PG sample of quasars. To check this the PG quasars in the sample of Wang et al. were isolated and the parameters of the straight line fit to these data are given in Table 1. These parameters are very similar to the parameters obtained for the current sample. The different slope obtained by Wang et al. is a consequence of the 'mix' of their sample.

Table 1. Correlation Parameters

\begin{tabular}{lccccc}
\hline & Ly $\alpha$ & C IV & \multicolumn{3}{c}{ H } \\
& IUE & IUE & PG & Boller/NLS & Wang/PG \\
\hline Num & 37 & 28 & 39 & 23 & 37 \\
Slope & $0.81 \pm 0.23$ & $-0.71 \pm 0.18$ & $-0.48 \pm 0.06$ & $0.58 \pm 0.15$ & $-0.52 \pm 0.07$ \\
SR & -0.22 & -0.38 & -0.73 & 0.14 & -0.67 \\
$P$ & 0.25 & $8.9 \times 10^{-2}$ & $1.1 \times 10^{-7}$ & 0.53 & $6.0 \times 10^{-7}$ \\
\hline
\end{tabular}

The strong correlation between the FWHM and the photon index for the low-ionization line $H \beta$ and the lack of this correlation for the high-ionization lines, Ly $\alpha$ and CIV, suggests that the regions emitting the low-ionization lines and the soft $\mathrm{X}$-rays may be causally linked in the PG sample of quasars. The lack of correlation for the narrow-line Seyfert 1 galaxies suggests that the distribution, geometry, and the kinematics of the $\mathrm{H} \beta$-emitting clouds in these AGNs are different from those in the PG quasars. The results presented in this paper suggest that the correlation between the FWHM of the $\mathrm{H} \beta$ line and the photon index of the soft $\mathrm{X}$-ray continuum and the differences in the correlation for different 'type' of AGNs are important diagnostics; this will be considered in detail in a separate publication (Gondhalekar, Rouillon-Foley, \& Kellett 1997).

\section{References}

Boller, Th., Brandt, W. N., \& Fink, H. 1996, A\&A, 305, 53.

Boroson, T.A., \& Green, R.F. 1992, ApJS, 80, 109.

Gondhalekar, P. M., Rouillon-Foley, C., \& Kellett, B. J. 1996a, A\&A, submitted. Gondhalekar, P. M., Rouillon-Foley, C., \& Kellett, B. J. 1996b, A\&A, submitted. Laor, A., Fiore, F., Elvis, M., Wilkes, B. J., \& McDowell, J. C. 1994, ApJ, 436, 611.

Wang, T., Brinkmann, W., \& Bergeron, J. 1996, A\&A, 309, 81. 